\title{
Transcurrencia a lo largo de la Falla Sierra de Varas (Sistema de fallas de la Cordillera de Domeyko), norte de Chile
}

\author{
Hans Niemeyer ${ }^{1}$, Carlos Urrutia ${ }^{2}$
}

\author{
${ }_{1}^{1}$ Departamento de Ciencias Geológicas, Universidad Católica del Norte, Casilla 1280, Antofagasta, Chile. \\ hansn@ucn.cl \\ 2 Exploraciones Mineras Andinas S.A., Avda. Apoquindo 4775, Of. 602, Las Condes, Santiago, Chile. \\ currutia@em.codelco.cl
}

RESUMEN. El Sistema de Fallas de la Cordillera de Domeyko, de rumbo esencialmente norte-sur, es paralelo a la fosa chileno-peruana en el norte de Chile. Se analizaron los desplazamientos en el rumbo y en la vertical de una de sus fallas maestras: la Falla Sierra de Varas (FSV). Ésta se estudió en el segmento entre Aguada del Hornito y Aguada del Cerro Alto de Varas, sobre la base del desplazamiento en el rumbo de dos porciones de la unidad de Granitoides paleozoicos con idéntica petrografía, estructura interna y edad. Se determinó una separación sinistral horizontal de 15,6 $\pm 1 \mathrm{~km}$ con una componente vertical de 4,9 $\pm 0,1 \mathrm{~km}$, lo cual implica un rechazo neto sinistral-inverso de $16,4 \pm 1 \mathrm{~km}$. El alzamiento vertical está de acuerdo con el espesor estratigráfico erosionado del bloque oriental. Se investigó, además, la dinámica y cinemática de la FSV mediante el análisis de mesofallas asociadas a sus desplazamientos. Se determinó así un sistema estructural sinistral-inverso, que tuvo lugar durante el Eoceno medio tardío. Un segundo sistema estructural dextral, con desplazamiento de $0,6 \mathrm{~km}$ se sobrepuso sobre el anterior y ocurrió con posterioridad al Mioceno. Los desplazamientos simultáneos, tanto en el rumbo como según fallas inversas, dados por la existencia de una 'flor inversa' en el segmento estudiado, indican que éste fue afectado primeramente por una transpresión. El desplazamiento sinistral demostrado para la FSV y su cambio de rumbo hacia el SE, inmediatamente al sur de Aguada de Alto de Varas, son compatibles con la vergencia hacia el oeste de pliegues y fallas inversas asociadas del cinturón plegado y corrido de El Profeta, el cual también habría resultado de la transpresión.

Palabras claves: Falla transcurrente, Mesofallas, Dinámica, Cinemática, Transpresión, Cordillera de Domeyko, Norte de Chile.

\begin{abstract}
Strike-slip along the Sierra de Varas Fault (Cordillera de Domeyko Fault-System), northern Chile. The north-south trending Cordillera de Domeyko Fault System in northern Chile considered herein is parallel to the Peru-Chile trench. The displacement history of the Sierra de Varas Fault, a master fault of the mentioned system, was examined in detail in the Aguada del Hornito-Aguada del Cerro Alto de Varas segment. Upper Paleozoic granitoids of the same composition, internal structure and age were cut and displaced by the fault. A sinistral horizontal separation of $15.6 \pm 1 \mathrm{~km}$ with a vertical component of $4.9 \pm 0.1 \mathrm{~km}$, suggests a sinistral-reverse net displacement of $16.4 \pm 1 \mathrm{~km}$. This is consistent with the local stratigraphic section that was eroded from the eastern block. A kinematic and dynamic analysis of mesofaults spatially related to the SVF displacements was conducted to identify the different fault populations and to obtain the stress tensor. Two structural systems were identified: an early reverse-strike-slip system and a late dextral superposed system. The first one ocurred during late middle Eocene, and the second is post-Miocene with an horizontal displacement of $0.6 \mathrm{~km}$. The presence of coeval strike-slip displacements along the Sierra de Varas Fault and reverse displacements in a 'reverse flower' in the studied segment show that the structural evolution of the Sierra de Varas was dominated by a bulk transpression during the late middle Eocene. The left-lateral displacement here demonstrated for the Sierra de Varas Fault and its inflection to the SE, south of the Aguada del Cerro Alto de Varas are compatible with the westward vergence of the folds and reverse faults in the El Profeta fault-and-thrust belt, which should be also the result of the transpression.
\end{abstract}

Keywords: Transcurrent fault, Mesofaults, Dynamics, Kinematics, Transpression, Cordillera de Domeyko, Northern Chile. 


\section{Introducción}

El Sistema de Fallas de la Cordillera de Domeyko (SFCD) tiene un rumbo general paralelo a la fosa chileno-peruana en el norte de Chile, de la cual dista unos $150 \mathrm{~km}$ al interior del continente (Fig. 1), por lo cual puede clasificarse como una 'trench-linked fault' según la terminología de Woodcock (1986). Se considera relacionado con un importante componente de transpresión ocurrido durante el Eoceno tardío-Oligoceno a lo largo de sus principales fallas (Mpodozis et al., 1993; Maksaev y Zentilli, 1999).

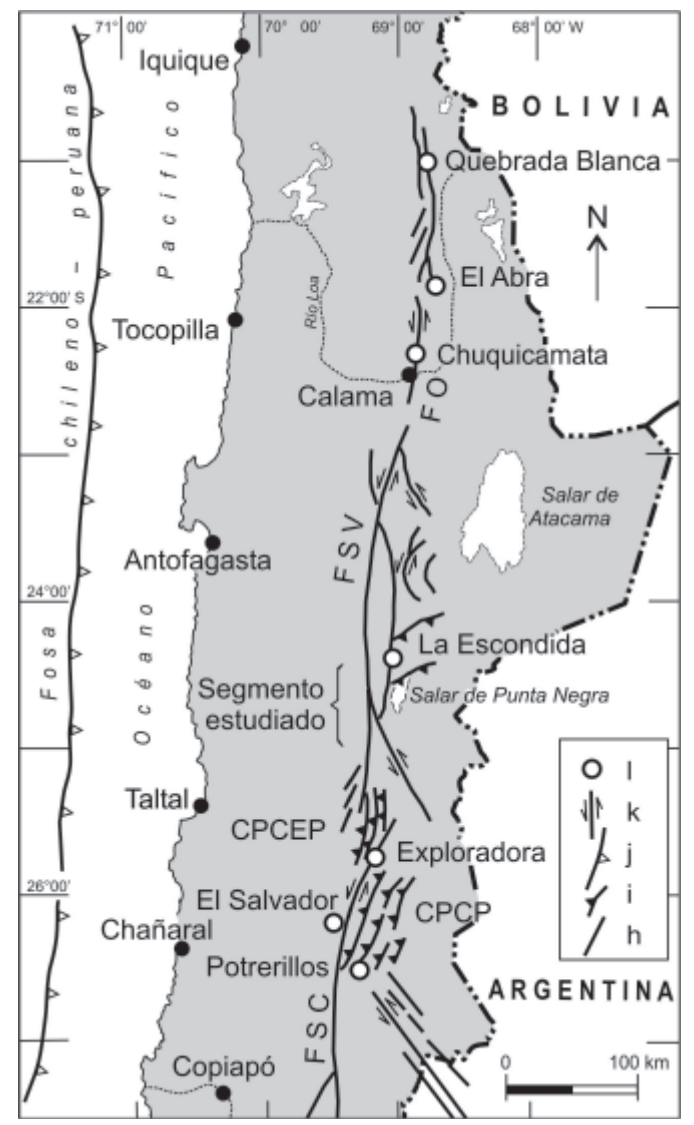

FIG. 1. El Sistema de Fallas de la Cordillera de Domeyko en el norte de Chile, según Cornejo y Mpodozis (1996'1). h. Fallas indiferenciadas; i. Fallas inversas; j. Fosa chilenoperuana; k. Fallas transcurrentes; l.Ubicación de pórfidos cupríferos; FO: Falla Oeste; FSV: Falla Sierra de Varas; FSC: Falla Sierra Castillo; CPCEP: Cinturón plegado y corrido de El Profeta; CPCP: Cinturón plegado y corrido de Potrerillos. Se indica el segmento de la Falla Sierra de Varas estudiado.
La Falla Sierra de Varas (FSV) es una estructura maestra del SFCD, extendiéndose por cerca de 300 $\mathrm{km}$ a lo largo de la Cordillera de Domeyko (Fig. 1), donde constituye el límite occidental de Sierra de Varas (Fig. 2a). Sin embargo, hasta el momento no se dispone de una estimación de la magnitud de sus desplazamientos, habiéndose sólo determinado un sentido de movimiento sinistral en su segmento norte (Mpodozis et al., 1993).

El propósito de esta contribución es determinar la magnitud de los desplazamientos de la FSV en el segmento Aguada del Hornito-Aguada de Cerro Alto de Varas, además de efectuar un análisis dinámico y cinemático. Lo primero se obtuvo utilizando como marcadores rocas plutónicas paleozoicas, así como también la estratigrafía de rocas sedimentarias paleozoicas, mesozoicas y cenozoicas involucradas en los bloques desplazados. Lo segundo se estableció sobre la base de la geometría, sentidos de desplazamientos y relaciones de corte de mesofallas, las que se consideran espacial, temporal y genéticamente ligadas con los desplazamientos de la FSV.

\section{Metodología de Trabajo}

Con el fin de estudiar los desplazamientos a lo largo de la FSV en el segmento Aguada del HornitoAguada de Cerro Alto de Varas, se aplicó a ella el concepto de 'plano de falla', el cual se asume vertical por presentar una traza rectilínea dentro de los límites de ese segmento. Para determinar la separación horizontal de la falla se utilizó el desplazamiento en el rumbo de dos porciones de granitoides paleozoicos que estuvieron juntas antes del funcionamiento de la falla, según se demuestra por su idéntica petrografía, estructura interna y edad. Para la determinación de la componente vertical del rechazo se siguieron dos líneas de evidencias independientes que, significativamente, resultaron ser concurrentes. La primera consistió en la utilización del 'rake' o barrido de mesofallas aledañas paralelas a la FSV, las cuales pueden ser asignadas a los mismos movimientos ocurridos a lo largo de la traza principal de la FSV, siguiendo el principio de que fallas transcurrentes paralelas tienen el mismo sentido de movimiento (Sylvester, 1988). La segunda consistió en la estimación del alzamiento tectónico de la Sierra de Varas, mediante la suma de los espesores estratigráficos de formaciones paleozoicas, mesozoicas y cenozoicas erosionadas del bloque oriental de la FSV. Final-

\footnotetext{
1 Geología de la región de Sierra Exploradora $\left(25^{\circ}-26^{\circ} \mathrm{S}\right)$. Servicio Nacional de Geología y Minería. Informe Registrado IR-96-09, 2 Vols. Santiago.
} 


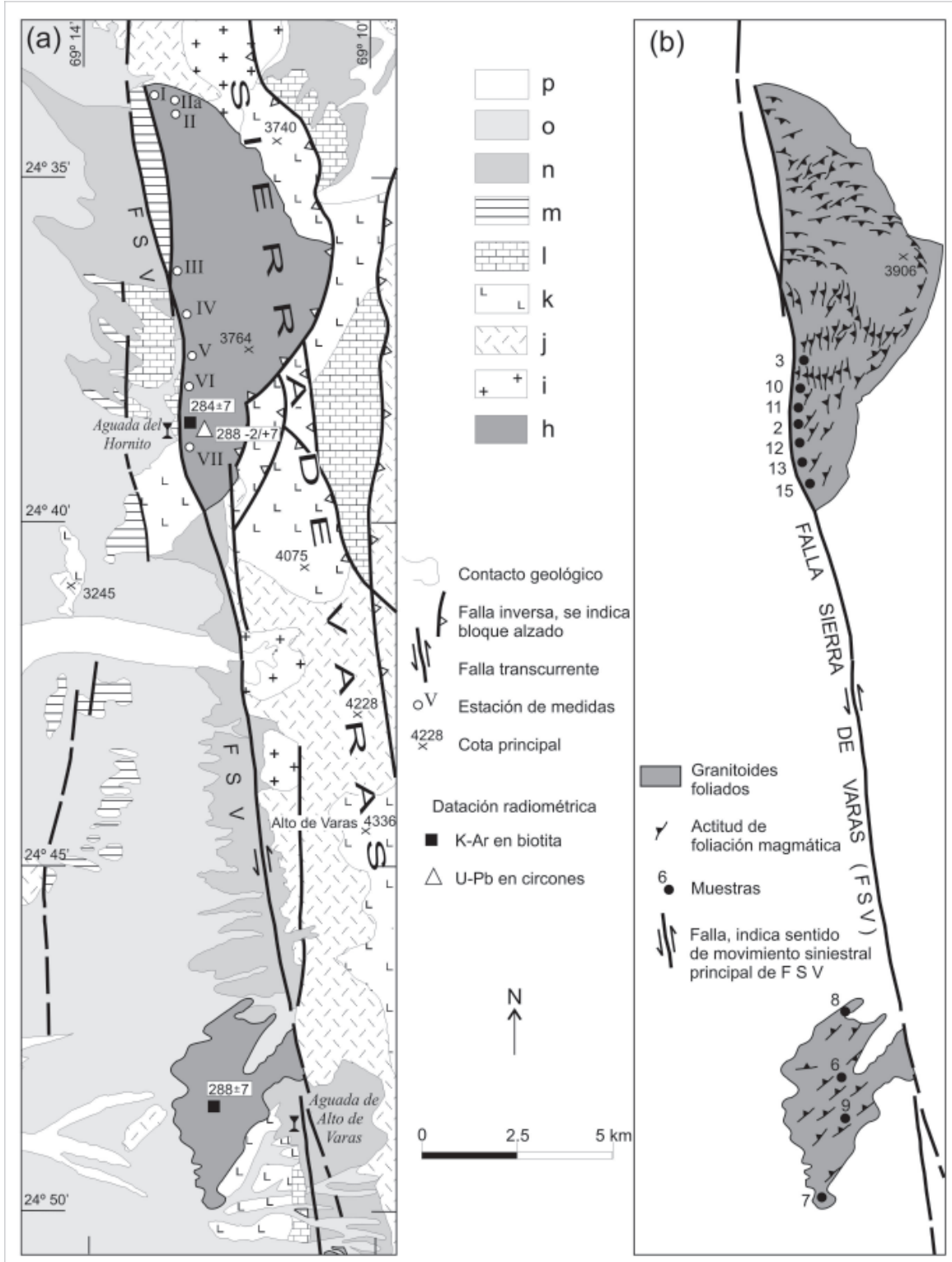

FIG. 2. (a) Mapa geológico de la Sierra de Varas en el área comprendida entre Aguada del Hornito y Aguada Cerro Alto de Varas (modificado de Hervé et al., 1991). h. Unidad de Granitoides foliados: tonalitas (Carbonífero superior); i. Granitoides no foliados: monzogranitos (Carbonífero-Pérmico); j. Intrusivos hipabisales: pórfidos dacíticos a riolíticos (CarboníferoPérmico); k. Formaciones La Tabla y Sierra de Varas: riolitas y lutitas calcáreas (Carbonífero); I. Formación EI Profeta: calizas, areniscas y lutitas calcáreas (Triásico Superior-Jurásico Superior); m. Formación Augusta Victoria: dacitas y andesitas (Paleoceno superior-Eoceno inferior); n. Formación Pampa de Mulas: gravas y areniscas semiconsolidadas (OligocenoMioceno); o. Depósitos aluviales y coluviales antiguos (Plioceno-Pleistoceno); p. Depósitos aluviales y coluviales modernos (Holoceno); (b) Granitoides foliados desplazados por la Falla Sierra de Varas. Las muestras se refieren a la clasificación petrográfica de la figura 4.

mente se calculó el rechazo aplicando Pitágoras. En este último cálculo se tomaron en cuenta los errores estimados en la determinación de los desplazamientos tanto horizontal como vertical y la propagación de éstos a través de la fórmula que da el valor de la hipotenusa de un triángulo rectángulo, según la metodología 'standard' de tratamiento de errores expuesta en Giamberardino (1989). 
El análisis dinámico y cinemático de mesofallas asociadas a la FSV se realizó en ocho estaciones ubicadas en el afloramiento de la unidad de Granitoides foliados de Aguada del Hornito (Fig. 2a). Esas rocas cumplen con las condiciones de ser isótropas, intactas y homogéneas, lo que las hace favorables para realizar tal análisis. La foliación de los granitoides no constituye una anisotropía respecto del fallamiento frágil, ya que ésta es de origen magmático y el macizo, como un todo, no se encuentra afectado en su comportamiento mecánico por la orientación mineralógica primaria. Se tiene, además, que la foliación magmática es interceptada con ángulos altos por las mesofallas asociadas a la FSV, lo cual indica que difícilmente ella podría controlar su posición, cuestión que -por lo demás- no se observa en terreno.

Sólo se consideraron en el análisis aquellas fallas con su datum completo, vale decir, fallas con rumbo y manteo, más 'rake' o barrido de la estría sobre el plano de falla, además de su sentido de movimiento definido por uno o más criterios cinemáticos según Petit(1987). Éstas se clasificaron según la geometría definida por los experimentos del tipo Riedel para fallas transcurrentes, tanto en modelos de arcilla (An y Sammis, 1996), arena (Naylor et al., 1986), así como también en experimentos en rocas (Bartlett et al., 1981). Especialmente, se tomó en cuenta el ángulo que forman las mesofallas con respecto a la traza de la FSV, además de su sentido de movimiento y relaciones de corte, clasificándolas como fallas secundarias del tipo R, R', P, Y, e inversas I, según nomenclatura de uso común (e.g., Woodcock y Schubert, 1994). Cabe destacar que las medidas de mesofallas, lejos de ser aleatorias, fueron tomadas mediante un criterio selectivo con el propósito de que ellas correspondieran lo más aproximadamente posible a mesofallas espacial, temporal y genéticamente ligadas con los desplazamientos de la FSV establecidos más arriba.

El tratamiento de los datos cinemáticos se realizó mediante el programa 'Stress' de Sperner et al. (1993), para DOS, que, como su nombre lo indica, se trata de un método dinámico, es decir, entrega la dirección de los 'stress' principales (Niemeyer, 2008). ¿Por qué utilizar un método dinámico para procesar datos cinemáticos? Esto se debe a que, ante la variedad de 'software' disponible, se llegó a la conclusión de que ese programa ofrece dos grandes ventajas. Primero, que al entregar la orientación de $\sigma_{1} \geq \sigma_{2} \geq \sigma_{3}$, relacionada con el conjunto de mesofallas introducido, compara los sentidos de movimiento de cada una de las mesofallas con el campo de 'stress' calculado para el total de datos ingresados. Cuando una falla no calza en orientación o sentido de desplazamiento con dicho campo de 'stress', es marcada como un 'valor negativo' por el programa. De esta manera, es posible desechar aquellas fallas geométricamente incompatibles con el campo de 'stress' establecido. Así se filtraron y revisaron los datos para todas las estaciones de observación utilizadas en este trabajo. Cabe señalar, que -en este caso- todos los datos introducidos al programa fueron, en realidad, compatibles con las direcciones de 'stress' establecidas para cada estación de medidas. Segundo, clasifica los datos sobre mesofallas en cuatro grados de confiabilidad establecidos por Hardcastle (1989), que van desde grado 1 (muy confiable) hasta grado 4 (poco confiable), otorgando mayor peso relativo a aquellas medidas con mayor grado de confiabilidad y restando importancia a aquellas con menor grado de confiabilidad.

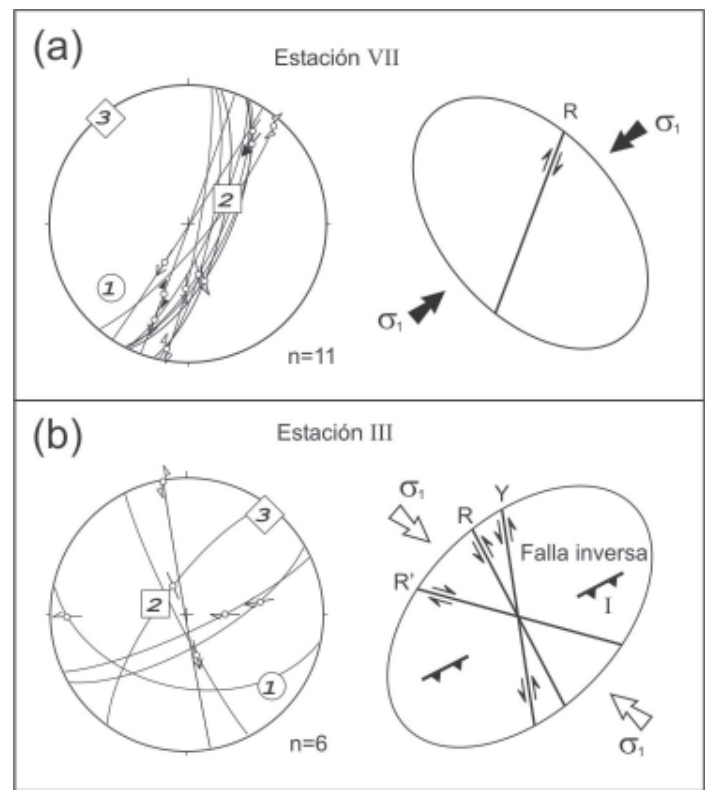

FIG. 3. Ejemplos de procesamiento de datos cinemáticos mediante el programa 'Stress' en dos estaciones de medidas. a. Estación de medidas VII; b. Estación de medidas III. A la izquierda, se muestra el análisis dinámico con el estereograma (proyección en red de Schmidt, hemisferio inferior) que incluye el datum de cada falla. Los números 1,2 y 3 indican respectivamente las direcciones de $\sigma_{1}$, $\sigma_{2}$ y $\sigma_{3}$. A la derecha, se indica el análisis cinemático de los mismos datos, con el dibujo de la elipse de 'strain' y la posición de las mesofallas, según nomenclatura de uso común (e.g., Woodcock y Schubert, 1994). 
A pesar de haber utilizado un programa dinámico para procesar los datos de mesofallas, se esbozó, para cada estación de medidas, la orientación de la elipse de 'strain' (análisis cinemático) tomando como base la distribución de los distintos tipos de mesofallas Riedel, procedimiento que es habitualmente empleado en la literatura geológico-estructural (e.g., Sylvester, 1988). Así, es posible también inferir cuáles fueron las direcciones de acortamiento y extensión máximas para cada estación. De este modo, se realiza simultáneamente un análisis dinámico y uno cinemático. En la figura 3 se muestra la aplicación dinámica del programa 'Stress' a dos estaciones de medidas, además de su interpretación cinemática.

\section{Características de la FSV en el segmento estudiado}

La FSV tiene un rumbo $\mathrm{N} 18^{\circ} \mathrm{O}$ y un manteo subvertical en el segmento comprendido entre la Aguada del Hornito y la Aguada de Cerro Alto de Varas, que se infiere por la forma de la traza de la falla en el terreno, la cual no se inflecta en las quebradas que la atraviesan, sino que es rectilínea.

En Aguada del Hornito, la FSV está dada por el contacto entre calizas, areniscas calcáreas y yesos de la Formación El Profeta del Triásico SuperiorJurásico (Chong, 1973) en el bloque occidental, y la unidad de Granitoides foliados (Smoje y Marinovic, 1994) del Carbonífero superior en el bloque oriental (Fig. 2a). A lo largo de la traza se observa, en afloramientos horizontales, una zona intensamente fracturada de $2-3 \mathrm{~m}$ de ancho con relleno de 'pseudocarniole', un tipo de roca de estructura cavernosa originada por la disolución de las calizas a consecuencia de la reacción química con yeso tectónicamente movilizado (Alberto et al., 2007).

En Aguada Cerro Alto de Varas, la FSV pone en contacto un afloramiento de Granitoides foliados, también del Carbonífero Superior (Padilla, 1988), en su bloque occidental con Pórfidos dacíticos a riolíticos (Carbonífero-Pérmico; Marinovic et al., 1995) en su bloque oriental (Fig. 2a). En ese lugar la falla se expone como una zona de cataclasita subvertical de 10-15 $\mathrm{m}$ de ancho. Tampoco aquí se observa un plano de falla definido, ni en el centro ni en los bordes de dicha zona. Por otro lado, se tiene que ella controla la hidrología del sector, provocando la surgencia de agua en las dos aguadas que definen el segmento estudiado (Herrera, 1995).

\section{Marcador del desplazamiento de la FSV}

Se infiere un movimiento transcurrente sinistral de la FSV por la separación de los dos afloramientos de Granitoides foliados del Carbonífero Superior mencionados arriba. El primero de ellos se ubica en Aguada del Hornito, al este de la FSV y, el segundo, en Aguada Cerro Alto de Varas, al oeste de dicha traza (Fig. 2b). A continuación se exponen tres argumentos que apoyan la hipótesis de que dichos afloramientos formaron parte de un mismo cuerpo con anterioridad a la actividad de la FSV.

Primero, se comparó su petrografía, tomando siete muestras del afloramiento de Aguada del Hornito y cuatro muestras del afloramiento de Aguada Cerro Alto de Varas (Fig. 2b). Se comprobó que ésta es idéntica y corresponde a tonalitas en ambos afloramientos. Esto surge de la comparación de conteos modales practicados en cortes transparentes de las muestras recolectadas (Fig. 4).

Segundo, se comparó su estructura magmática. Se constató que ambos afloramientos presentan una foliación magmática, de orientación similar, anterior al desplazamiento de la FSV. De este modo, en el sector sur del afloramiento de Aguada del Hornito la fábrica foliada del granitoide tiene un rumbo NE con manteos al NO, la cual se trunca contra la FSV. En Aguada Cerro Alto de Varas la foliación tiene la misma actitud general que en Aguada del Hornito (Fig. 2b). Esto sugiere que el lugar de mejor correspondencia del afloramiento de Aguada Cerro Alto de Varas es con la porción meridional del afloramiento de Aguada del Hornito.

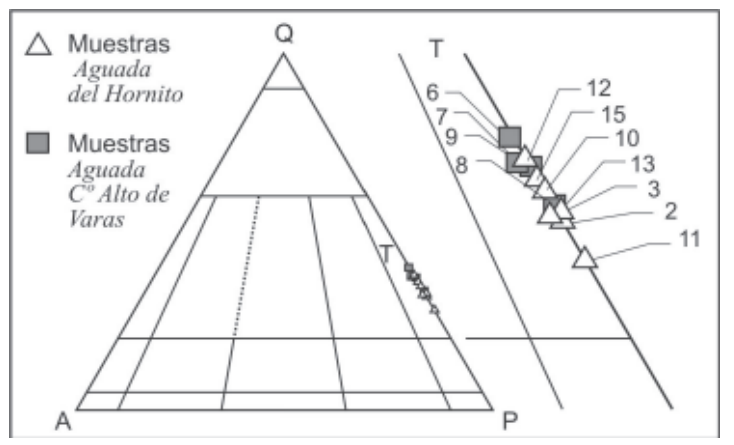

FIG. 4. Clasificación de los Granitoides foliados del Carbonífero Superior procedentes de la Aguada del Hornito y la Aguada Cerro Alto de Varas en el triángulo QAP (Q: cuarzo; A: feldespato alcalino; P: feldespato plagioclasa; T: campo de las tonalitas) de Streckeisen (1974). 
Tercero, se compararon las edades de ambos afloramientos de la unidad de Granitoides foliados disponibles en la literatura. Se comprobó que son concordantes dentro de su error analítico, más aún si fueron hechas por el mismo método y en el mismo mineral. Efectivamente, en Aguada del Hornito se dispone de una edad K-Ar de $284 \pm 7$ Ma en biotita (Smoje y Marinovic, 1994), que se homologa con otra edad K-Ar de 288 \pm 7 Ma también en biotita procedente de Aguada Cerro Alto de Varas (Padilla, 1988). Debe agregarse que la edad radiométrica de los afloramientos de granitoides de Aguada del Hornito se ubica en la porción sur del afloramiento, que es la que se correlaciona con el afloramiento de Aguada Cerro Alto de Varas. En esa misma porción se localiza además una edad convencional U-Pb en grupos de circones de 288-2/+7 Ma (Marinovic et al., 1995) que también es concordante, dentro de su error analítico, con las otras dos edades (Fig. 2a). Lo anterior indica que las edades K-Ar no acusan rejuvenecimiento y que, por lo tanto, se están comparando dos porciones de roca plutónica que se emplazaron y enfriaron al mismo tiempo.

\section{Rechazo de la FSV}

Un desplazamiento sinistral de la FSV en el segmento Aguada del Hornito-Aguada Cerro Alto de Varas se basa en la separación horizontal de los respectivos límites septentrionales de los afloramientos de Granitoides paleozoicos (Fig. 2b). El error de la separación se estima en $\pm 1 \mathrm{~km}$ ( $6 \%$ de error relativo; Portuondo, 1988), quedando, por lo tanto, la sepa-

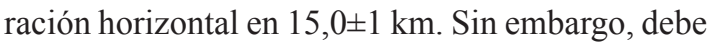
sumarse a este valor el desplazamiento relacionado con una posterior reactivación posmiocena de 0,6 $\mathrm{km}$ en sentido dextral de la falla (Herrera, 1995), y se obtiene una separación horizontal anterior al

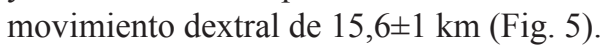

Además, es necesario explicar el alzamiento vertical del bloque oriental en Aguada del Hornito, donde sólo afloran rocas paleozoicas, respecto del bloque occidental, en el cual se exponen rocas mesozoicas y cenozoicas. Se utilizó el 'rake' o barrido de mesofallas aledañas paralelas a la FSV, las cuales se asignan a los mismos movimientos ocurridos a lo largo de la traza principal de la FSV (ver Metodología de trabajo). Así, se obtiene como promedio aritmético $17^{\circ} \mathrm{S}$ para el 'rake' o barrido de esas fallas (Tabla 1).
TABLA1. DATUMDE OCHO MESOFALLAS EN POSICIÓN' 'Y' PARALELAS A LA FALLA SIERRA DE VARAS.

\begin{tabular}{cccc}
\hline Estación & Posición & Rake & Movimiento \\
\hline II & $\mathrm{N} 3^{\circ} \mathrm{O} / 40^{\circ} \mathrm{SO}$ & $25^{\circ} \mathrm{S}$ & sinistral \\
III & $\mathrm{N} 10^{\circ} \mathrm{O} / 89^{\circ} \mathrm{SO}$ & $12^{\circ} \mathrm{SE}$ & sinistral \\
V & $\mathrm{NS} / 55^{\circ} \mathrm{E}$ & $0^{\circ}$ & sinistral \\
V & $\mathrm{N} 7^{\circ} \mathrm{O} / 80^{\circ} \mathrm{SO}$ & $0^{\circ}$ & sinistral \\
VI & $\mathrm{N} 7^{\circ} \mathrm{O} / 55^{\circ} \mathrm{NE}$ & $20^{\circ} \mathrm{SE}$ & sinistral \\
VI & $\mathrm{N} 3^{\circ} \mathrm{O} / 55^{\circ} \mathrm{NE}$ & $20^{\circ} \mathrm{SE}$ & sinistral \\
VI & $\mathrm{NS} / 54^{\circ} \mathrm{E}$ & $15^{\circ} \mathrm{S}$ & sinistral \\
VI & $\mathrm{N} 5^{\circ} \mathrm{O} / 52^{\circ} \mathrm{NE}$ & $42^{\circ} \mathrm{SE}$ & sinistral \\
& & & \\
& & & \\
& & & \\
\hline
\end{tabular}

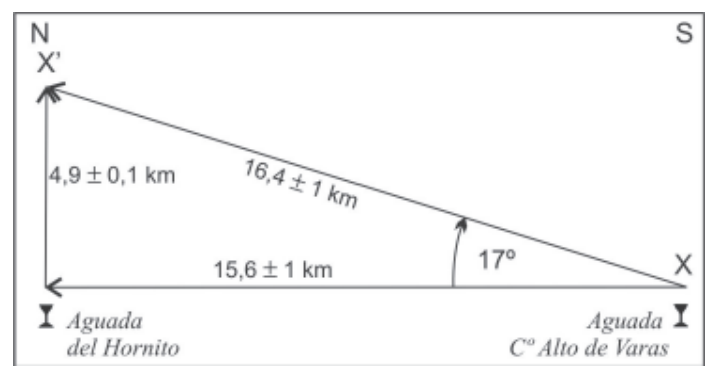

FIG. 5. Vectores componentes del desplazamiento sinistral-inverso de la Falla Sierra de Varas, con su error estimado. El error en la cuantificación del rechazo obedece a la propagación de los errores de los catetos al calcular el valor de la hipotenusa según Pitágoras.

Como ya quedó dicho, el valor del 'rake' de $17^{\circ} \mathrm{S}$ se puede extrapolar al 'plano de falla' de la FSV. Por lo tanto, es posible reconstruir un rechazo sinistralinverso para ella. De este modo se determina, para la separación horizontal de 15,6 $1 \mathrm{~km}$, un alzamiento del bloque oriental de la FSV de 4,9 km (Fig. 5). Más abajo se determina el error en la estimación de dicho alzamiento, sobre la base de consideraciones estratigráficas.

Una manera independiente de estimar el rechazo vertical de la FSV consiste en sumar los espesores de rocas estratificadas que fueron erosionadas del bloque oriental de la FSV en el sector de Aguada del Hornito. En efecto, según Marinovic et al. (1995), los espesores de esas rocas son: Formación La Tabla (0,8-1,0 km), Formación Sierra de Varas $(0,1 \mathrm{~km})$, Formación El Profeta $(2,1$ km), Formación Santa 
Ana $(0,5 \mathrm{~km})$, Formación Augusta Victoria $(1,0 \mathrm{~km})$ y Formación Pampa de Mulas (0,3-0,4 km). Consecuentemente, la suma de los espesores de rocas paleozoicas, mesozoicas y cenozoicas erosionadas al este de la FSV da como resultado un mínimo de $4,8 \mathrm{~km}$ y un máximo de $5,1 \mathrm{~km}$. Se constata, por lo pronto, que el alzamiento estimado mediante el 'rake' queda comprendido entre esos dos valores extremos. Esto significa que, como máximo, se puede tolerar un error de $\pm 0,1 \mathrm{~km}$ en la estimación del rechazo vertical ( $2 \%$ de error relativo; Portuondo, 1988). Por lo tanto, el rechazo vertical de la falla puede cuantificarse en un mínimo de 4,9 $9 \pm 0,1$ km (Fig. 5), ya que la cúpula del plutón paleozoico expuesto en Aguada del Hornito se encuentra erosionada en su mayor parte (González, 2007).

En consecuencia, aplicando el teorema de Pitágoras al triángulo de la figura 5 , se obtiene un rechazo neto sinistral-inverso de $16,35 \pm 0,98 \mathrm{~km}$, una vez estudiada la propagación de los errores individuales a través de la fórmula que da el valor de la hipotenusa. Luego, aproximando, se tiene que la magnitud del rechazo neto sinistral-inverso es de $16,4 \pm 1 \mathrm{~km}$.

\section{Análisis dinámico y cinemático}

En lo que sigue se efectúa un análisis dinámico y cinemático de mesofallas asociadas a los desplazamientos de la FSV. Se identifican dos sistemas estructurales asociados a la transcurrencia de la FSV: sistema sinistral-inverso y sistema dextral.

\subsection{Sistema estructural sinistral-inverso}

Corresponde al sistema de fallas mejor desarrollado en el segmento de FSV estudiado. Mesofallas relacionadas con este sistema se encuentran en las estaciones I, II, IIa, III, IV, V y VI. Allí se logró realizar una cantidad de 75 medidas de fallas con su datum completo. En este sistema se desarrollan claramente distintas mesofallas secundarias: fallas $\mathrm{R}$, fallas R', fallas Py fallas inversas I, además de fallas $\mathrm{Y}$, paralelas a la traza de la FSV. Es característico de este sistema el desarrollo de fallas inversas I de rumbo NE (Estaciones III, IV y VI). Las respectivas mesofallas de este sistema se ilustran en la figura 6, donde, además de su posición, se indica la de la estría y el movimiento del bloque colgante para cada una de ellas.

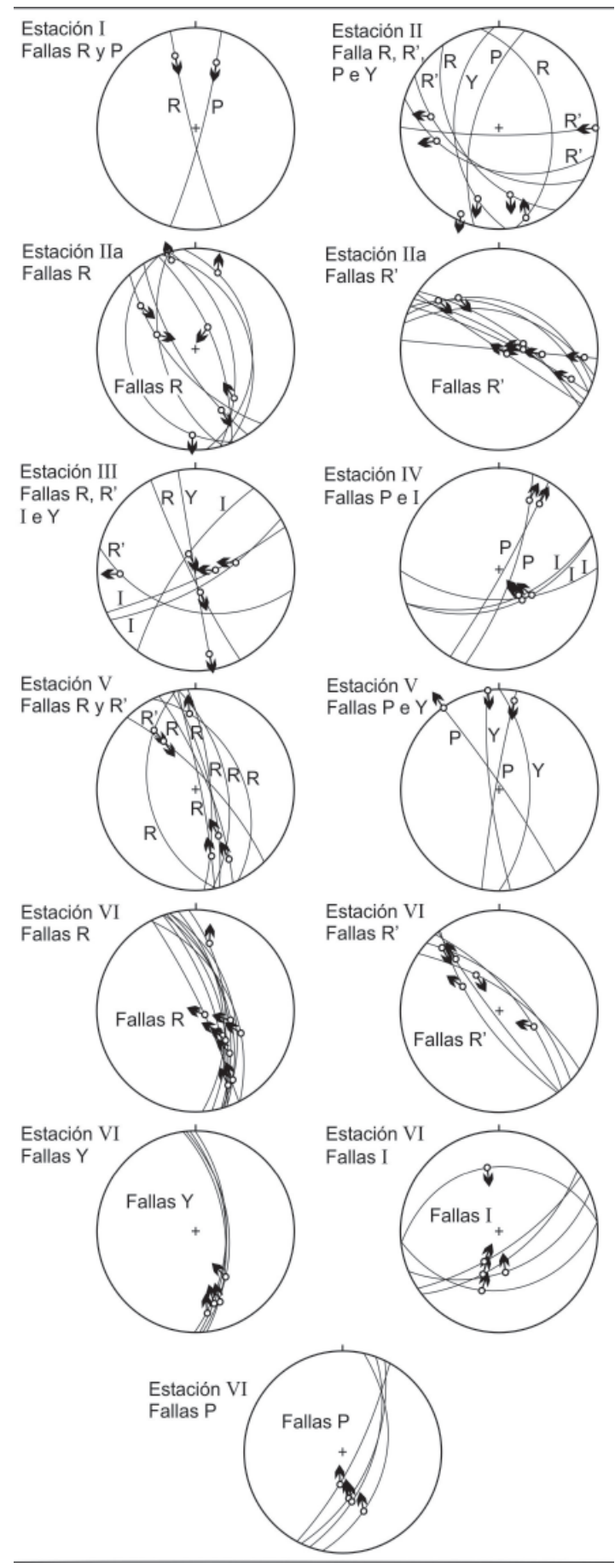

FIG. 6. Mesofallas del sistema sinistral-inverso. Se indica la estría y el movimiento del bloque colgante para cada una de las fallas, además del valor cinemático asignado a ellas según nomenclatura de uso común (e.g., Woodcock y Schubert, 1994). Proyección en red de Schmidt, hemisferio inferior. 
La estación I corresponde a un afloramiento de mala calidad donde sólo fue posible identificar un par de fallas en posición R y P. En la estación II los datos corresponden a mesofallas en posición R, R', $\mathrm{P}$ e Y. La estación IIa se sitúa sobre una zona con cataclasis antigua que no se relaciona con la FSV (Niemeyer et al., 2004), donde las mesofallas nuevas más comunes son las R' seguidas de las $\mathrm{R}$. La estación III presenta mesofallas en posición $R, R^{\prime}$ e Y, además de fallas inversas I. En la estación IV se observan fallas en posición P y fallas inversas I. En la estación V se observan mesofallas del tipo R, R', P e Y, así como también se observan fracturas $\mathrm{T}$ con rellenos de calcita de 5-7 $\mathrm{mm}$ de espesor. En la estación VI están muy bien desarrolladas las mesofallas, entre las cuales las más abundantes son las R (Fig. 7), seguidas de las R', P e Y, con algunas fallas en posición inversa $\mathrm{I}$.

El resultado del análisis dinámico de este sistema se indica en la columna izquierda de la figura 8 , donde, por medio de flechas negras, se dibujan las respectivas direcciones de $\sigma_{1}$, las que ocupan posiciones variables $\mathrm{NNO}$ a $\mathrm{NO}$, entregadas por el programa 'Stress', desde la Estación I a la Estación VI. En particular, se observa que las fallas inversas I resultan ser perpendiculares a la dirección de $\sigma_{1}$ obtenida del análisis dinámico. Además, se indican esquemáticamente las respectivas elipses de 'strain' inferidas de la orientación y cinemática de las mesofallas para cada estación.

\subsection{Sistema estructural dextral}

Corresponde a un sistema con menor expresión que el sistema sinistral-inverso, por cuanto la cantidad de mesofallas es menor. El sistema se encuentra representado en las estaciones II, III, IV, V, VI y VII, donde se realizaron 44 medidas de mesofallas con su datum completo. Las respectivas mesofallas de este sistema se ilustran en la figura 9, donde, además de su posición, se indica la posición de la estría y el movimiento del bloque colgante para cada una de ellas.

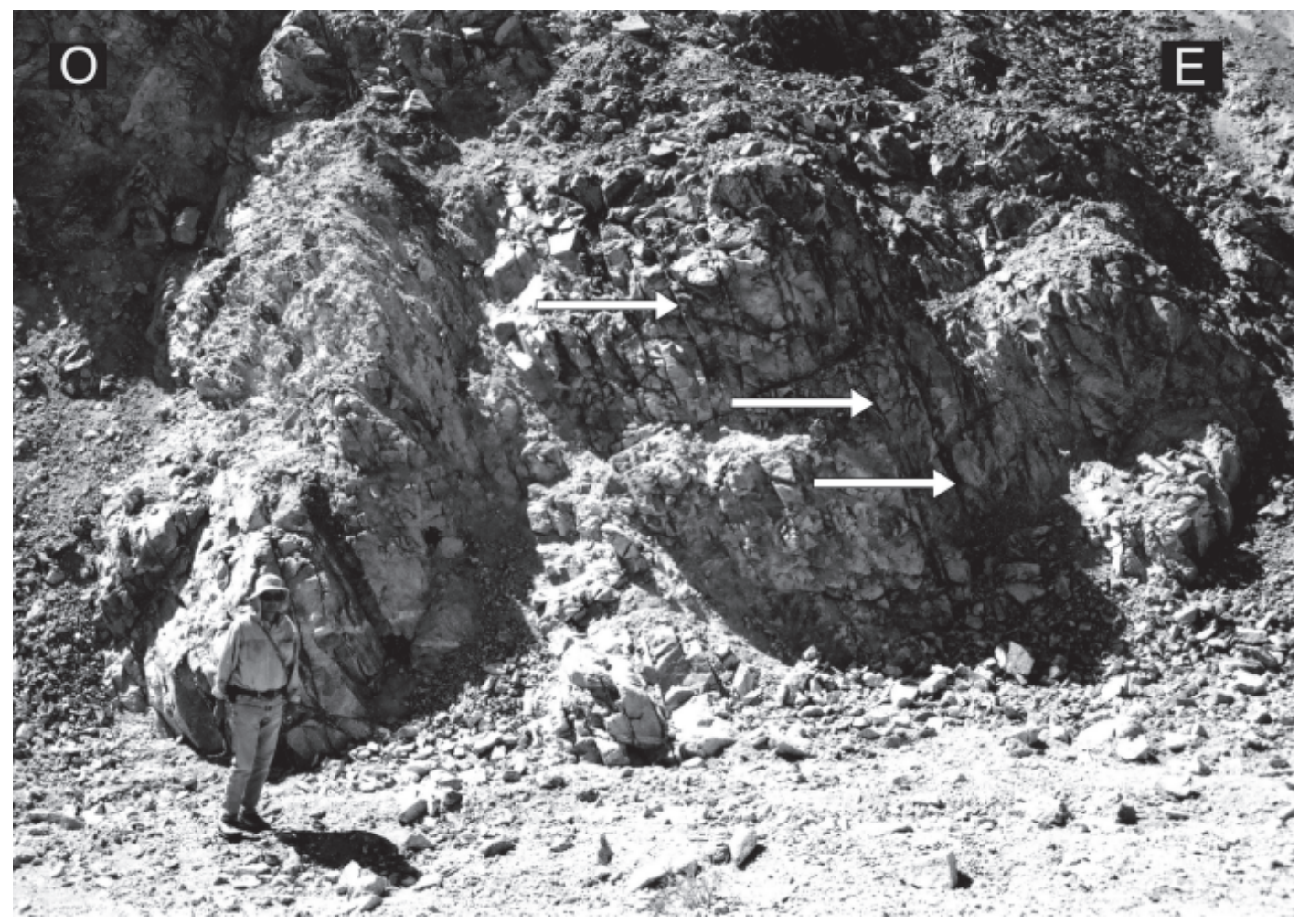

FIG. 7. Mesofallas del sistema sinistral-inverso en posición R. Afloramiento vertical en la Estación VI. Las flechas indican algunas de las trazas de mesofallas en el afloramiento. 


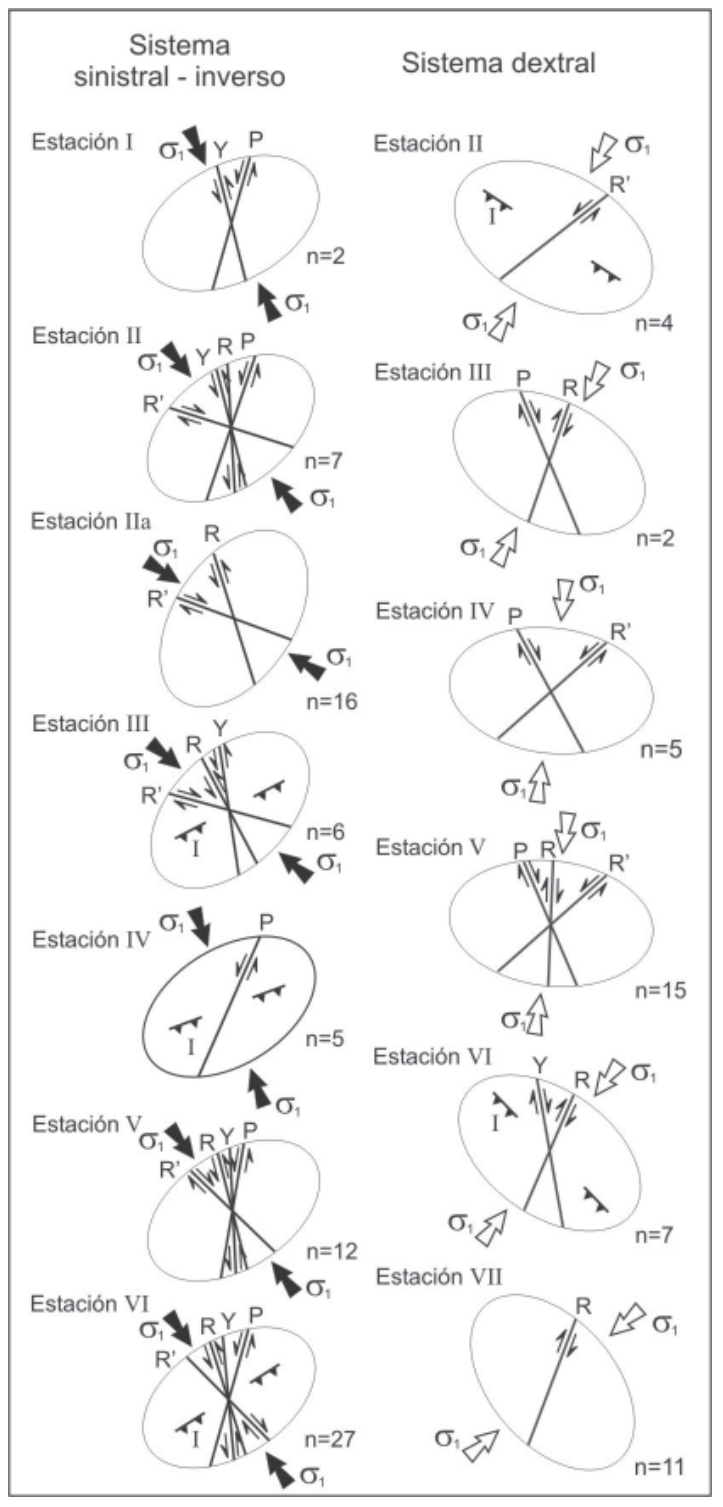

FIG. 8. Análisis cinemático de mesofallas asociadas a la Falla Sierra de Varas según sistema sinistral (columna del lado izquierdo) con flechas negras indicando la dirección de $\sigma_{1}$, y sistema dextral (columna del lado derecho) con flechas blancas indicando la dirección de $\sigma_{1}$. Se esboza también, para cada estación, la elipse de 'strain' deducida de las distintas posiciones de las mesofallas.

Las direcciones mejor expuestas de este sistema son las fallas $\mathrm{R}$ y las fallas $\mathrm{P}$, mientras que aquellas en posición R' se desarrollan de una manera menor. También se asocian fallas inversas de orientación NO. En la estación II se registran mesofallas en

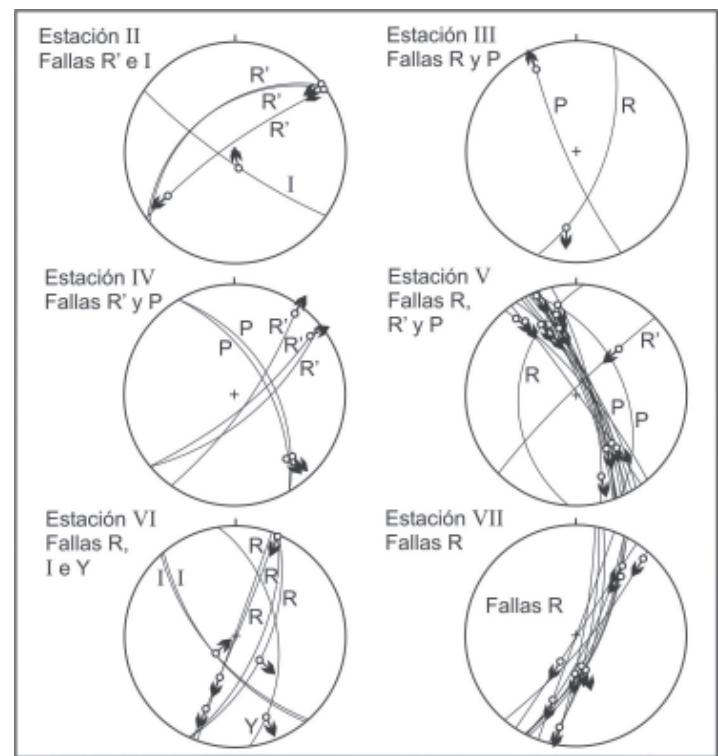

FIG. 9. Mesofallas del sistema dextral. Se indica la estría y el movimiento del bloque colgante para cada una de las fallas, además del valor cinemático asignado a cada una de ellas según nomenclatura de uso común (e.g., Woodcock y Schubert, 1994). Proyección en red de Schmidt, hemisferio inferior.

posición R'e inversa. En la estación III se observan escasas fallas en posición $\mathrm{R}$ y P. La estación IV exhibe mesofallas R'y P, donde las fallas más claramente desarrolladas son las de posición $\mathrm{P}$, con orientación NNO. En la estación V se observan fallas R' de rumbo NE y abundantes fallas $\mathrm{P}$ en posición NNO (Fig. 10). En la estación VI predominan las fallas en posición $R$, pero también se observó una falla $Y$, además de otra falla inversa I. En la estación VII sólo se observaron abundantes fallas en posición R.

El resultado del análisis dinámico de este sistema se ilustra en la columna derecha de la figura 8 , donde, por medio de flechas blancas, se dibujan las respectivas direcciones de $\sigma_{1}$, las que ocupan posiciones variables NNE a NE, entregadas por el programa 'Stress', desde la Estación II a la Estación VII. Además, se indican esquemáticamente las respectivas elipses de 'strain' inferidas de la orientación y cinemática de las mesofallas para cada estación.

El hecho de que fallas sinistrales de rumbo NNO (en posición $\mathrm{R}$ del sistema sinistral-inverso) se observen cortadas y desplazadas por fallas dextrales NNE (en posición R del sistema dextral) es indicativo de que el sistema dextral sucedió en el tiempo al 


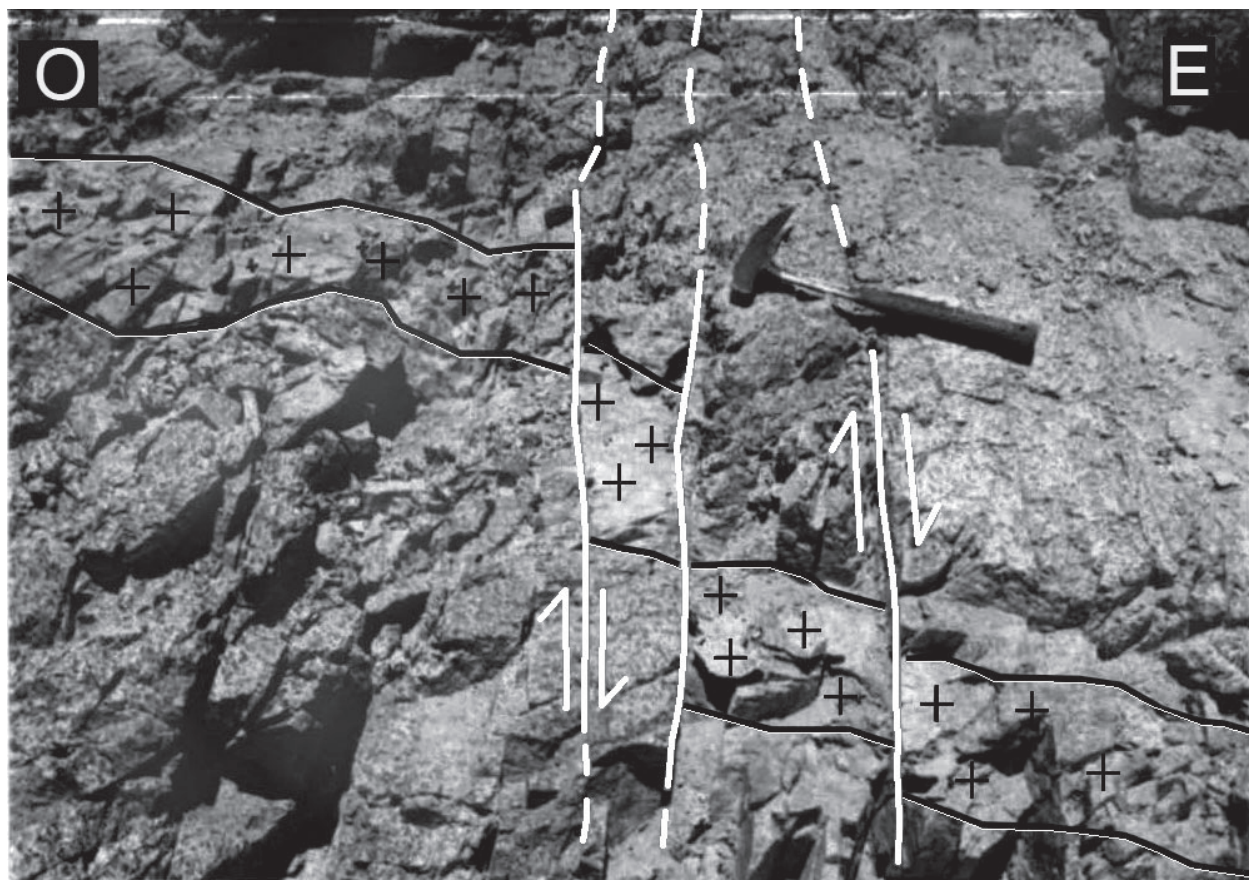

FIG. 10. Mesofallas del sistema dextral en posición P resaltadas en blanco. Desplazan a dique aplítico, resaltado con cruces. Mediante semiflechas se indica el sentido de movimiento a lo largo de las mesofallas. Afloramiento horizontal en Estación V.

sistema sinistral-inverso, lo cual concuerda con los datos regionales arriba expuestos. Otra característica importante de este sistema es que reactiva fracturas $\mathrm{T}$ del movimiento sinistral-inverso antiguo, quedando estas fracturas en una posición cercana a las fallas $\mathrm{P}$, respecto al sistema dextral.

Una forma independiente de determinar el movimiento dextral tardío y su dirección de $\sigma_{1}$ asociada, es mediante la orientación de pliegues 'chevron' centimétricos y asimétricos con vergencia al NE en capas de calizas finamente laminadas de la Formación El Profeta en la Estación VII (Fig. 11). Se observa que la dirección de $\sigma_{1}$ determinada por este método es coincidente con la determinada sobre la base del análisis cinemático de mesofallas en la misma estación (ver figura 8).

\section{Discusión}

En el segmento estudiado se han descrito estructuras contemporáneas de transcurrencia sinistral, dadas por la FSV (McElderry et al., 1997), y acortamiento, dado por la existencia de una 'flor inversa' ubicada $3 \mathrm{~km}$ al NE de Aguada del Hornito (Hervé et al., 1991) (Fig. 2a). Esta última se caracteriza

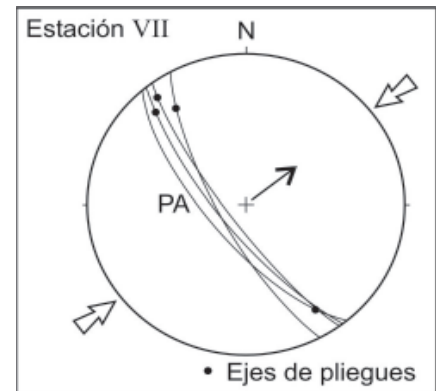

FIG. 11. Ejes de pliegues 'chevron' asimétricos y sus planos axiales (PA). La flecha delgada indica la vergencia de los pliegues hacia el NE, en tanto que las flechas gruesas blancas indican la dirección de $\sigma_{1}$. Proyección en red de Schmidt, hemisferio inferior.

por estar conformada por fallas inversas de rumbo norte-sur y vergencia opuesta, y que la limitan por el oeste y por el este, dejando entre medio una zona estrecha de rocas de la Formación Sierra de Varas intensamente deformadas (Fig. 2a). Lo anterior constituye una evidencia de que el desplazamiento sinistral-inverso de la FSV se encuentra estrechamente relacionado con una transpresión. 
La dificultad mayor que presenta el estudio de las fallas de rumbo es que, generalmente, no se dispone de marcadores fehacientes para establecer la magnitud de sus movimientos (Sylvester, 1988). Esto se debe a que ellas poseen desplazamientos paralelos al rumbo de la estratificación en las rocas sedimentarias que, en el caso de la Sierra de Varas, están materializadas principalmente por las capas triásico-jurásicas de la Formación El Profeta (Chong, 1973). Es precisamente en estas secuencias, en que las fallas inversas pueden ser más notorias al realizar perfiles estructurales este-oeste, donde ciertos autores han llegado a la conclusión errónea de que sólo ocurre una tectónica compresional en la Cordillera de Domeyko (Amilibia, 2001; Amilibia y Skarmeta, 2003). Sin embargo, el desplazamiento sinistral demostrado aquí para la FSV y su cambio de rumbo hacia el SE, inmediatamente al sur de la Aguada de Alto de Varas, es compatible con la vergencia hacia el oeste de pliegues y fallas inversas del Cinturón plegado y corrido de El Profeta (CPCEP, Fig. 1; Chong y Reutter, 1985). Esto último sugiere que ese cinturón es también el resultado de una transpresión sinistral. Al respecto se puede agregar que, más al sur, la relación causa-efecto entre el desplazamiento sinistral de varios kilómetros de la Falla de Sierra Castillo (FSC, Fig. 1) y el Cinturón plegado y corrido de Potrerillos (CPCP, Fig. 1) está fehacientemente demostrada (Tomlinson et al., 1993; Tomlinson et al., 1994). Se enfatiza aquí, por lo tanto, la idea de que fallas inversas y fallas de rumbo no son excluyentes. Por el contrario, son coetáneas. Efectivamente, ellas -en conjunto- dan cuenta de un ambiente tectónico transpresivo.

Aunque no se dispone de un elemento cronológico directo que permita fijar la edad de la transpresión, se tiene que el pórfido de La Escondida, con una edad

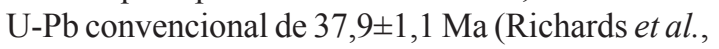
1999) y ubicado $40 \mathrm{~km}$ al norte, dentro de un lente de cizalle del SFCD (Mpodozis et al., 1993), se emplazó dentro de un ambiente tectónico transpresivo sinistral (Padilla et al., 2001). Por consiguiente, se puede sugerir que la actividad tectónica transpresiva sinistral se habría producido durante el Eoceno Medio Tardío en el segmento analizado.

Finalmente, debe agregarse que la presencia de clastos procedentes de las diferentes formaciones, tanto paleozoicas como mesozoicas de la Sierra de Varas dentro de la Formación Pampa de Mulas (Marinovic et al., 1995), indica que el relieve tectónico principal de dicha Cordillera ya estaba construido en el Mioceno. De este modo, es posible relacionar directamente el alzamiento de la Sierra de Varas con el régimen tectónico transpresivo que en ella imperó durante el Eoceno medio tardío.

\section{Conclusiones}

La FSV presenta dos eventos de movimientos transcurrentes: uno que ha quedado registrado por un desplazamiento sinistral-inverso de la unidad de Granitoides foliados del Carbonífero Superior (Smoje y Marinovic, 1994), el cual se desarrolló durante el Eoceno Medio Tardío, y el otro, por un desplazamiento posterior al Mioceno, de carácter dextral. El desplazamiento sinistral-inverso tiene una componente horizontal de $15,6 \pm 1 \mathrm{~km}$, con una importante componente en la vertical de 4,9 $9,1 \mathrm{~km}$. Este último valor surge de dos líneas de evidencias independientes: una a partir del 'rake' o barrido de $\sim 17^{\circ} \mathrm{S}$ atribuido a la estría de su 'plano de falla', el cual se asume vertical, y la otra, a partir de la erosión de las sucesiones estratigráficas de rocas paleozoicas, mesozoicas y cenozoicas de su bloque oriental. Se llega así a un rechazo compuesto sinistral-inverso de $16,4 \pm 1 \mathrm{~km}$. La cinemática de ambos eventos ha quedado marcada, respectivamente, en dos sistemas estructurales de mesofallas secundarias dentro de la unidad de Granitoides foliados del Carbonífero Superior. El primer evento dejó mesofallas muy marcadas en las siguientes posiciones: R, R', P y fallas inversas I, además de fallas $\mathrm{Y}$, paralelas a la traza de la FSV. El análisis dinámico entregó posiciones de $\sigma_{1}$ variables entre NNO y NO para este evento. El segundo evento de transcurrencia se superpuso sobre el anterior, originando un movimiento dextral de $0,6 \mathrm{~km}$, con posterioridad al Mioceno. Este último se expresa en mesofallas superpuestas que, en parte, reactivaron fracturas del desplazamiento anterior, correspondientes al sistema sinistral-inverso. El análisis dinámico entregó posiciones de $\sigma_{1}$ variables entre NNE y NE para este último evento. En forma independiente, para una de las estaciones de medidas, se estimó la posición de $\sigma_{1}$ sobre la base de la geometría y vergencia de pliegues centimétricos 'chevron' en calizas finamente laminadas, que coincidió con esa misma dirección, establecida por el análisis dinámico de mesofallas.

\section{Agradecimientos}

Los autores agradecen las observaciones de J. Cembrano (Departamento de Ciencias Geológicas, Universi- 
dad Católica del Norte), que contribuyeron a mejorar esta publicación. Asimismo, agradecen las observaciones de un corrector anónimo y de A. Rodríguez Perea (Universitat de les Illes Balears, España). El financiamiento de las campañas de terreno se debe a la Dirección de Investigaciones y Postgrados de la Universidad Católica del Norte. L. Jofré (Departamento de Ciencias Geológicas, Universidad Católica del Norte) confeccionó los dibujos para esta publicación.

\section{Referencias}

Alberto, W.; Carraro, F.; Giardino, M.; Tiranti, D. 2007. Genesis and evolution of 'pseudocarniole': preliminary observations from the Susa Valley (Western Alps). In Evaporites through space and time (Schreiber, B.; Lugli, C.; Ba, Bel, M.; editors). Geological Society, London, Special publication 285: 155-168.

Amilibia, A. 2001. Inversión tectónica en la Cordillera de Domeyko, Andes del Norte de Chile. Tesis de Doctorado (Inédito), Universidad de Barcelona, Departamento de Geodinámica y Geofísica: 149 p.

Amilibia, A.; Skarmeta, J. 2003. La inversión tectónica en la Cordillera de Domeyko en el norte de Chile y su relación con la intrusión de sistemas porfíricos de Cu-Mo. In Congreso Geológico Chileno, No. 10, Actas, Sesión Temática 2: 1-7. Concepción.

An, L.-J.; Sammis, C.G. 1996. Development of strike-slip faults: shear experiments in granular materials and clay using a new technique. Journal of Structural Geology 18 (8): 1061-1077.

Bartlett, W.L.; Friedman, M.; Logan, J.L. 1981. Experimental folding and faulting of rocks under confining pressure, Part IX. Wrench faults in limestone layers. Tectonics 79: 255-277.

Chong, G. 1973. Reconocimiento geológico del área Catalina-Sierra de Varas y estratigrafía del Jurásico del Profeta, Provincia de Antofagasta. Memoria de Título (Inédito), Universidad de Chile, Departamento de Geología: 284 p.

Chong, G.; Reutter, K.J. 1985. Fenómenos de tectónica compresiva en las Sierras de Varas y de Argomedo, Precordillera chilena, en el ámbito del paralelo $25^{\circ}$ sur. In Congreso Geológico Chileno, No. 4, Actas: 219- 238. Antofagasta.

González, R. 2007. Mecanismo de emplazamiento del Plutón Sierra de Varas, Cordillera de Domeyko, Norte de Chile. Tesis de Doctorado (Inédito), Universidad Católica del Norte, Departamento de Ciencias Geológicas: $222 \mathrm{p}$.

Giamberardino, V. 1989. Teoría de los errores. Editorial Reverté Venezolana S.A.: 168 p. Caracas.

Hardcastle, K.C. 1989. Possible paleostress tensor configurations derived from strike-slip data in eastern Vermont and western New Hampshire. Tectonics 8 (2): 265-284.

Herrera, C . 1995. Geología e hidrogeología del área Sierra de Varas-Sierra Vaquillas Altas (2430' $-25^{\circ} 30^{\prime}$ ' de latitud sur y $69^{\circ} 00^{\prime}-69^{\circ} 30^{\prime}$ de longitud oeste). Memoria de título (Inédito), Universidad Católica del Norte, Departamento de Ciencias Geológicas: 137 p.

Hervé, M.; Marinovic, N.; Mpodozis, C.; Smoje, I. 1991. Mapa Geológico de la Hoja Sierra de Varas (1:100.000), Región de Antofagasta. Servicio Nacional de Geología y Minería, Documento de Trabajo 2.

Maksaev, V.; Zentilli, M. 1999. Fission Track Thermochronology of the Domeyko Cordillera, Northern Chile: Implications for Andean Tectonics and Porphyry Copper Metallogenesis. Exploration and Mining Geology, Special Issue on Latin American Mineral Deposits 8 (1-2): 65-89.

Marinovic, N.; Smoje, I.; Maksaev, V.; Hervé, M.; Mpodozis, C. 1995. Hoja Aguas Blancas, Región de Antofagasta. Servicio Nacional de Geología y Minería, Carta Geológica de Chile 70. Santiago.

McElderry, S.; Prior, D.J.; Chong-Díaz, G.; Potts, G.S.; Flint, S. 1997. Kinematics of the West Fissure Fault System. In Congreso Geológico Chileno, No. 8, Actas 3: 684-688. Antofagasta.

Mpodozis, C.; Marinovic, N.; Smoje, I. 1993. Eocene left lateral strike slip faulting and clockwise block rotations in the Cordillera de Domeyko, west of Salar de Atacama, northern Chile. In International Symposium on Andean Geodynamics (ISAG), No. 2, Actas: 25-228. Oxford, United Kingdom.

Naylor, M.A.; Mandl, G.; Sijpesteinjn, C.H.K. 1986. Fault geometries in basement-induced wrench faulting under different initial stress states. Journal of Structural Geology 8: 737-752.

Niemeyer, H. 2008. Geología Estructural. Editorial RIL: 292 p. Santiago.

Niemeyer, H.; Berríos, H.; Ruiz-Cruz, M.D. 2004.Temperaturas de formación en cataclasitas triásicas de la Cordillera de Domeyko, Antofagasta, Chile. Revista Geológica de Chile 31 (1): 3-18.

Padilla, H. 1988. Eventos intrusivos y deformaciones en la Cordillera de Domeyko a la latitud del Salar de Punta Negra: Antecedentes geocronológicos K-Ar. In Congreso Geológico Chileno, No. 5, Actas 3: I229I243. Santiago.

Padilla, R.A.; Titley, S.R.; Pimentel, F. 2001. Geology of the Escondida Porphyry Copper Deposit, Antofagasta Region, Chile. Economic Geology 96: 307- 324.

Petit, J. 1987. Criteria for the sense of movement on fault surfaces in brittle rocks. Journal of Structural Geology 9: 593-608.

Portuondo, R. 1988. Procesamiento de datos experimentales. Editorial ENPE: 292 p. La Habana.

Richards, J.P.; Noble, S.P.; Pringle, M.S. 1999. A Revised Late Eocene Age for Porphyry Cu Magmatism in the Escondida Area, Northern Chile. Economic Geology 94: 1231-1248.

Smoje, I.; Marinovic, N. 1994. Intrusivos del Carbonífero-Pérmico en la Cordillera de Domeyko: Nuevos 
antecedentes radiométricos. In Congreso Geológico Chileno, No. 7, Actas 2: I213-I216. Concepción.

Sperner, B.; Ratschbacher, L.; Ott, R. 1993. Fault-striae analysis: a turbo pascal program package for graphical presentation and reduced stress tensor calculation. Computer and Geosciences 19 (9): 1361-1388.

Streckeisen, A. 1974. Rocas plutónicas. Clasificación y nomenclatura recomendada por la Unión Internacional de Ciencia Geológicas (IUGS). Subcomisión en sistemática de rocas ígneas. Instituto de Investigaciones Geológicas (Inédito-Traducción al castellano de Neues Jahrbuch für Mineralogie, Abhandlungen 107: 144-240).

Sylvester, A.G. 1988. Strike-slip faults. Geological Society of America Bulletin 100: 1666-1703.

Tomlinson, A.J.; Mpodozis, C.; Cornejo, P.; Ramírez, C.F 1993. Structural Geology of the Sierra Castillo-

Manuscrito recibido: abril 13, 2007; aceptado: julio 8, 2008.
Agua Amarga Fault System, Precordillera of Chile, El Salvador-Potrerillos. In International Symposium on Andean Geodynamics (ISAG) No. 2, Actas: 259262. Oxford, United Kingdom.

Tomlinson, A.J.; Mpodozis, C.; Cornejo, P.; Ramírez, C.F.; Dumitru, T. 1994. El Sistema de fallas Sierra Castillo-Agua Amarga: transpresión sinistral eocena en la Precordillera de Potrerillos-El Salvador. In Congreso Geológico Chileno, No. 7, Actas 2: 14591463. Concepción.

Woodcock, N.H. 1986. The role of strike-slip fault systems at plate boundaries. Philosophical Transactions of the Royal Society A317: 13-29. London.

Woodcock, N.H.; Schubert, C. 1994. Continental Strike-Slip Tectonics. In Continental Deformation (Hancock, P.L.; editor), Pergamon Press: 421 p. 